\title{
Flying Car Transportation System: Advances, Techniques, and Challenges
}

\author{
Gaofeng Pan, SeniorMember, IEEE, and Mohamed-Slim Alouini, Fellow, IEEE
}

\begin{abstract}
In order to fulfill transportation demands, people have well-explored ground, waterborne, and high-altitude spaces (HAS) for transportation purposes, as well as the underground space under cities (namely, subway systems). However, due to the increased burdens of population and urbanization in recent decades, huge pressures on public transportation and freight traffic are introduced to cities, plaguing the governors and constraining the development of economics. By observing the fact that near-ground space (NGS) has rarely been utilized, researchers and practitioners started to re-examine, propose and develop flying cars, which are not a totally novel idea, aiming at solving the traffic congestion problem and releasing the strains of cities. Flying cars completely differ from traditional grounded transportation systems, where automobiles/trains are suffered track limitations and are also different from the air flights in HAS for long-distance transfer. Therefore, while observing the lack of specific literature on flying cars and flying car transportation system (FCTS), this paper is motivated to study the advances, techniques, and challenges of FCTS imposed by the inherent nature of NGS transportation and to devise useful proposals for facilitating the construction and commercialization of FCTS, as well as to facilitate the readers understanding of the incoming FCTS. We first introduce the increased requirements for transportation and address the advantages of flying cars. Next, a brief overview of the developing history of flying cars is presented in view of both timeline and technique categories. Then, we discuss and compare the state of the art in the design of flying cars, including take-off \& landing (TOL) modes, pilot modes, operation modes, and power types, which are respectively related to the adaptability, flexibility \& comfort, stability \& complexity, environment friendliness of flying cars. Additionally, since largescale operations of flying cars can improve the aforementioned transportation problem, we also introduce the designs of FCTS, including path and trajectory planning, supporting facilities and commercial designs. Finally, we discuss the challenges which might be faced while developing and commercializing FCTS from three aspects: safety issues, commercial issues, and ethical issues.

Index Terms-Autonomous pilot, commercial design, commercialization, environment friendliness, flying car transportation system, freight delivery, human transportation, near-ground space, safety, traffic congestion.
\end{abstract}

\section{INTRODUCTION}

Along with the fast increase of population, cities across the world are growing rapidly in the physical dimension in the past few decades, especially cities in some developing and developed countries, like the ones in Europe and Asia. The increasing level of urbanization has brought about significant

Manuscript received**, 2019; revised **, 2019; accepted **, 2019. The associate editor coordinating the review of this paper and approving it for publication was ***. (Corresponding author: Gaofeng Pan.)

G. Pan and M.-S. Alouni are with Computer, Electrical and Mathematical Sciences and Engineering Division, King Abdullah University of Science and Technology (KAUST), Thuwal 23955-6900, Saudi Arabia. city and business boom, leading to high geographical functionalization of city modules. Therefore, huge pressures on public transportation and freight traffic have also been introduced at the same time with the economic developments, which conversely suffocate the further development of both society and economy.

So far, humans have well-explored ground, waterborne, and high-altitude space (HAS), and explored underground space (namely, subway) to fulfill our transportation demands, as shown in Fig. 1. As shown in Table I, comparisons among the main transportation systems are presented based on geographical spaces. It is easy to see that the most popular grounded transportation systems, i.e., road/train transport, are suffered track limitations, resulting in poor flexibility and congestion while the load is high, especially in urban areas. Moreover, nowadays, waterborne transport, including maritime and fluvial transports, is mainly used for long-distance freight transport in view of its low transport cost, thus it is not suitable for urban transport. Also, the HAS transportation system, namely, air transportation, is usually for long-distance human/goods delivery with high cost, leading to impracticality for urban applications. Therefore, it can be concluded from Table I that only underground, grounded and NGS transports are suitable for urban scenarios.

Therefore, intuitively, in order to remove these obstacles on the way of development, various schemes have been proposed and implemented by the governors of the cities and related researchers to improve and optimize the existing underground and grounded transportation systems, e.g., metro and public bus systems, and further to enlarge the traffic load and to fulfill the increasing traffic demands. However, obviously, there exist maximum limitations of the traffic loads for the underground and grounded transportation systems. Then, for the sake of essentially elevating the traffic loads, some researchers and engineers resort to the unexplored near-ground space (NGS) for help, the attitude of which ranges from tens to hundreds of meters ${ }^{1}$, and then flying cars are accordingly re-examined, proposed and developed.

Observing the lack of specific literature on flying cars and flying car transportation system (FCTS), the main purposes of this article are to propose an introduction, review, and discussion on the past, present, and future of FCTS from various aspects. Namely, we will respectively elaborate on the development history of flying cars, the designs of flying cars and FCTS, and the challenges and prospects of FCTS, thus

\footnotetext{
${ }^{1}$ In fact, up to now, NGS has rarely been used, except for limited helicopter and unmanned aerial vehicles (UAV).
} 


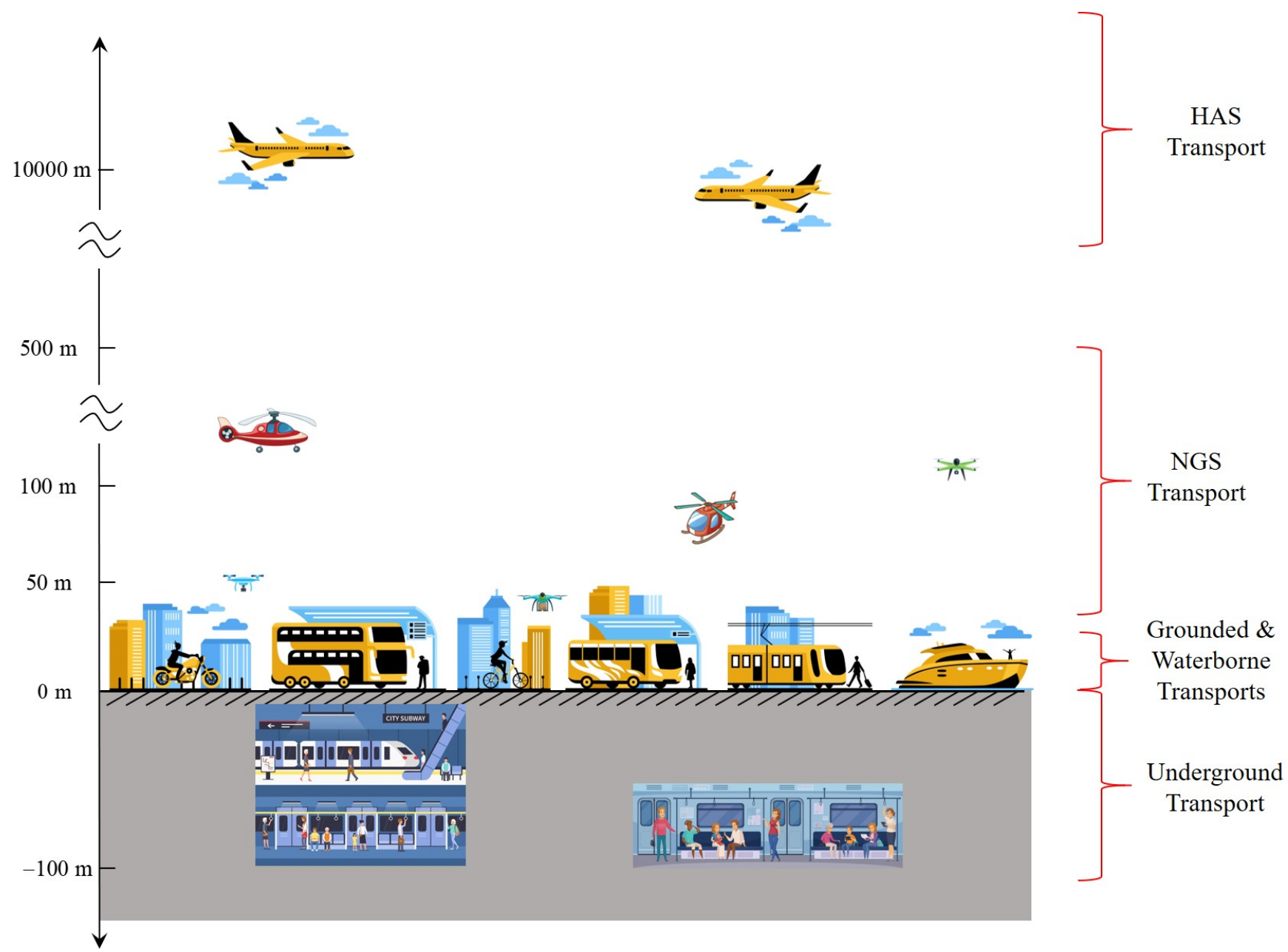

Fig. 1: Main transportation systems.

TABLE I: Comparisons Among The Main Transportation Systems

\begin{tabular}{|c|c|c|c|c|c|c|}
\hline \hline Types & Distance & Objects & Cost & Flexibility & Load & Utilization status \\
\hline Underground transport & Short & People & High & Poor & Large & Explored \\
\hline Grounded transport & All covered & People/Goods & Medium & Medium & Medium & Well explored \\
\hline Waterborne transport & Long & Goods & Low & Poor & Large & Well explored \\
\hline NGS transport & Short & People/Goods & Low & Good & Small & Rarely explored \\
\hline HAS transport & Long & People/Goods & High & Good & Small & Well explored \\
\hline \hline
\end{tabular}

showing a comprehensive picture of FCTS to facilitate the readers to understand FCTS.

In the following parts of this section, we first introduce the notation of FCTS, and then talk about the necessity of developing FCTS. Finally, a brief description of the organization of the remainder of this article will be presented.

\section{A. What is FCTS?}

Differing from traditional transportation systems, as a novel transportation system that makes use of flying cars to exploit the NGS, FCTS is capable of expanding public/private transportation to increase traffic load and accelerate the movements of humans and goods, especially in urban areas, while simultaneously reducing traffic congestion and air pollution.
Therefore, compared with existing transportation systems, the core advantage of FCTS is the revolutionary step of systematically and commercially exploiting the NGS for the movement of humans and goods, though helicopters have already been developed to utilize the same space. Specifically, in FCTS, flying cars will operate and be organized under systematic control to provide fast and convenient but affordable human and freight transfer services for both public and private purposes.

\section{B. Why Should FCTS Be Introduced?}

In this subsection, we will answer the question that why FCTS should be introduced for future cities. Alternatively speaking, the benefits brought to the society by FCTS will be discussed. 
As mentioned in the previous subsection, FCTS flying cars are used to transfer people or goods from one place to another while operating in the NGS. Consequently, some essential facilities for traditional ground-based transportation systems, e.g., the common infrastructure components (roads and tunnels), can be totally avoided and saved in FCTS. Furthermore, electric-powered rotors are designed and equipped with flying cars to reduce the on-board noise and emissions quite dramatically, and further, make the travel experience more comfortable. Finally, flexible operation mechanisms can be implemented and launched in FCTS, thanks to the freedom arising from the inherent system characteristics of FCTS.

To clearly address the advantages of FCTS, some unique properties of FCTS can be summarized as follows:

1) Environment-friendly transportation is supported, as power cells are equipped with flying cars and zero discharge can be theoretically achieved;

2) Congestion free transportation is available, benefiting from the rarely unexploited and unlimited NGS resource;

3) Flexible and fast door-to-door transportation is obtained, as shorter travel paths can be realized by flying cars to make the same journey compared with on-ground transportation systems;

4) Less ground supporting infrastructures are required, as most of the operation behaviors occur in the near ground airspace and vertical take-off \& landing (VTOL) mode has been considered and adopted as the main and popular mode for flying cars;

5) Competitions among all kinds of road users can be reduced, and then more on-ground space can be freed up for people, making cities more clear and comfortable;

6) Less construction and upkeep costs are needed to build up and run FCTS, as no roads or tunnels are required to construct and maintain, leading to low-cost commercial operations for FCTS.

Observing these aforementioned advantages, we can conclude that FCTS can serve as a promising solution to relieve traffic congestion in cities and promote the process of urbanization at a low cost.

\section{The Architecture of This Article}

The rest of this paper is organized as follows. In Section II, we present a brief review of the developing history of flying cars. In Section III and IV, the designs of flying cars and FCTS are elaborated, respectively. The challenges and trends of FCTS are discussed and summarized in Section V. Finally, the paper is concluded with some remarks in Section VI.

\section{The Developing History of Flying Cars}

In fact, flying car is not a novel idea and can be traced back to the attempts made by the people in the early of last century. In this section, a brief introduction to the developing history of flying cars will be presented by following both timeline and technique categories.

In the early 1900s, the airplane had been successfully invented and manufactured, and the automobile was further

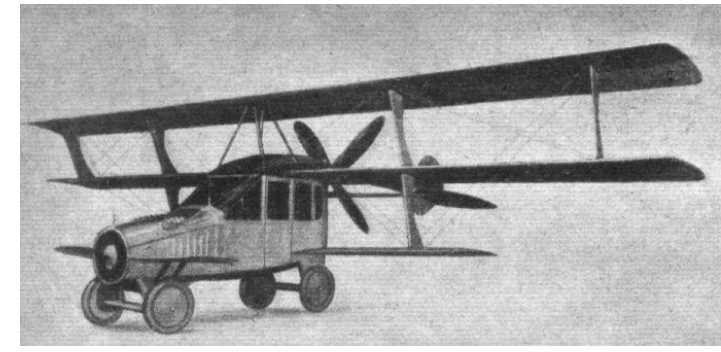

Fig. 2: Curtiss' Aeroplane in 1917. (https://upload.wikimedia. org/wikipedia/commons/b/b6/Curtiss_Autoplane_1917.jpg.)

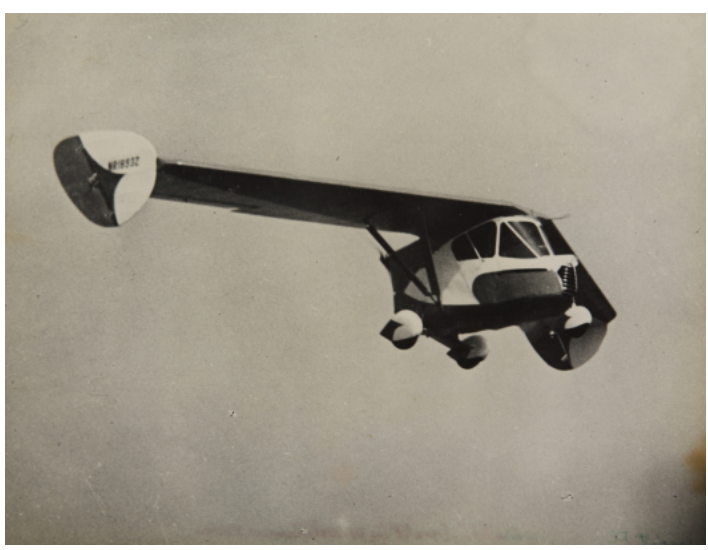

Fig. 3: The Waterman Aerobile in 1937. (https: //en.wikipedia.org/wiki/Waterman_Arrow-bile\#/media/File: Waterman_Aerobile_in_flight.jpg.)

perfected and massively produced by profiting from the development of the automotive industry (namely, standard massproduction techniques). Then, people tried to combine airplane and motorcar for the sake of merging the advantages of them, like predicted by Henry Ford in 1940: "Mark my word: a combination of airplane and motorcar is coming. You may smile, but it will come" [1]. Following this technical road-map, endeavors were made in succession to find out suitable and practical engineering proposals, e.g., Glen Curtiss' Aeroplane debuted in 1917 [2], the Waterman Aerobile in 1937 [3], the ConVairCar Model 118 in 1947 [4], the Aero-Car series from 1949 to 1977 [5], [6], and the AVE Mizar in 1973 [7].

Observing these prototypes of flying cars shown in Figs. 26 , there is one thing in common that, in essence, fixed wings are equipped to enable automobiles to take-off, fly and land in these designs.

Meanwhile, in the last century, there also existed another technical branch which tried to build up flying cars by making use of duct fans to realize VTOL behaviors, e.g., the Volante Tri-Athodyne in 1956-1958 by Ford [8], Piasecki's VZ-8 Airgeep in 1958-1962 by Piasecki Aircraft [9], and the Sky Commuter in the 1980s by Fred Barker [10].

Unfortunately, the attempts of the aforementioned two kinds of technical flows to establish flying cars in last century were all failed, not only because of the immaturity of the related technologies at that time but also due to the fact that the relatively advanced and expensive designs are beyond the practical needs and affordability of the consumers in last 


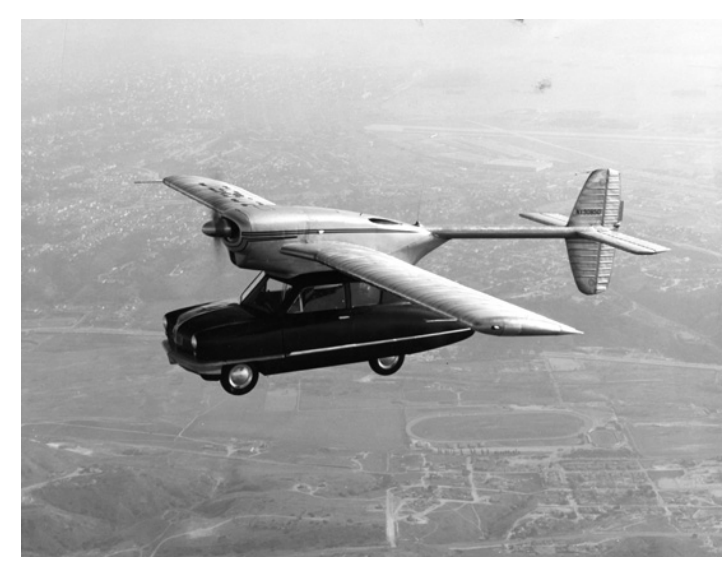

Fig. 4: The ConVairCar Model 118 in 1947. (https://upload. wikimedia.org/wikipedia/commons/thumb/b/b5/ConvairCar_ Model_118.jpg/450px-ConvairCar_Model_118.jpg)

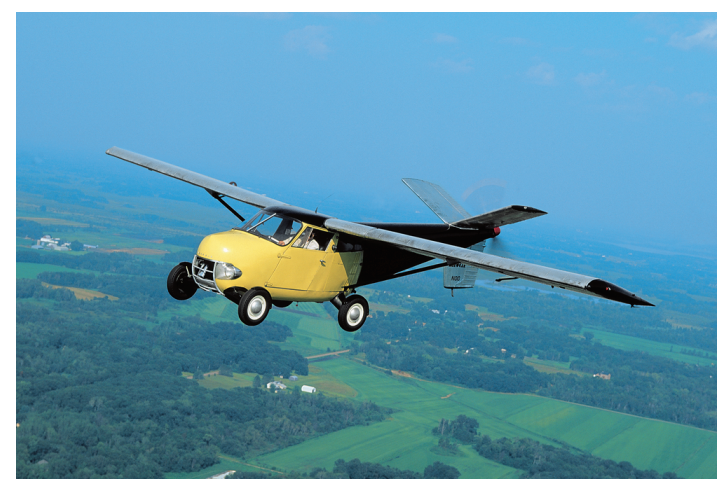

Fig. 5: Aero-Car Model I in 1954. (https://barrettjacksoncdn. azureedge.net/staging/carlist/items/Fullsize/Cars/236076/ 236076_Front_3-4_Web.jpg)

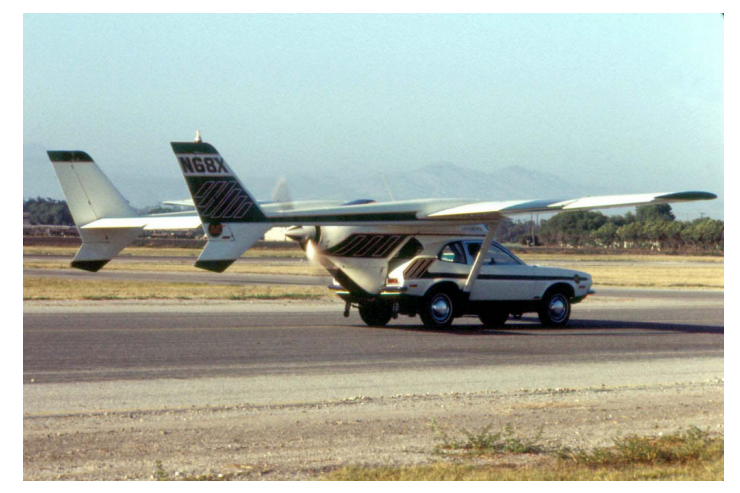

Fig. 6: The AVE Mizar in 1973. (https://upload.wikimedia.org/ wikipedia/commons/thumb/8/80/AVE-Mizar-1973-N68X-XL. jpg/450px-AVE-Mizar-1973-N68X-XL.jpg)

century.

In this new century, profiting from the speedy advancements of power batteries, metallic and non-metallic materials, automatic control, and mechanical manufacturing, the development of flying cars has been greatly broadened. Also, the motivation of the commercialization of flying cars has been greatly enhanced, due to the increased transportation

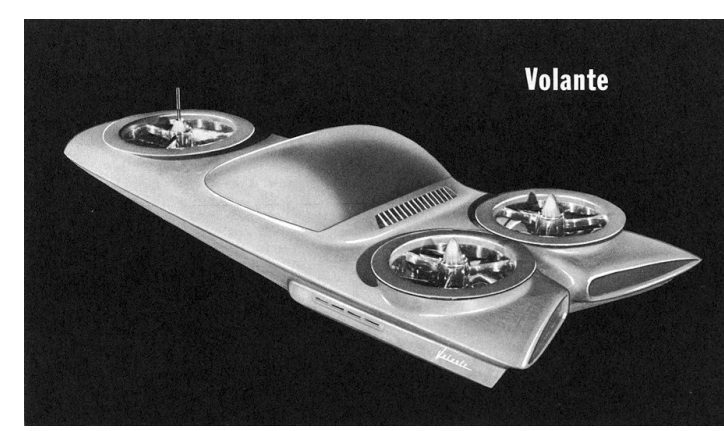

Fig. 7: The Volante Tri-Athodyne in 1957. (http://www.carstyling.ru/resources/concept/ 1957-Ford-Volante-Concept-Car-Model-01.jpg)
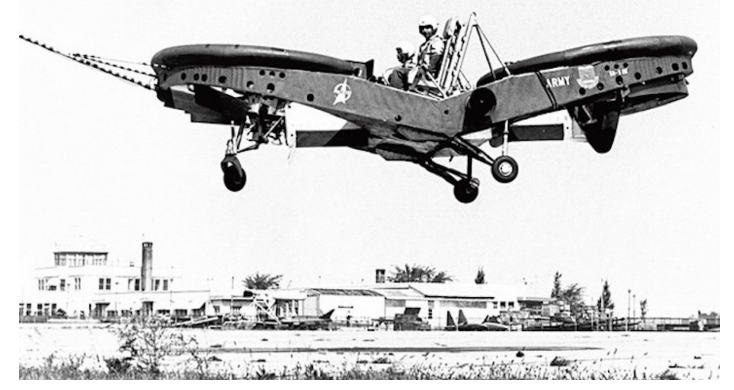

Fig. 8: Piasecki's VZ-8 Airgeep in 1962. (https://cdn.motor1. com/images/mgl/XJP9E/s1/the-armys-quest-for-a-flying-jeep. jpg)

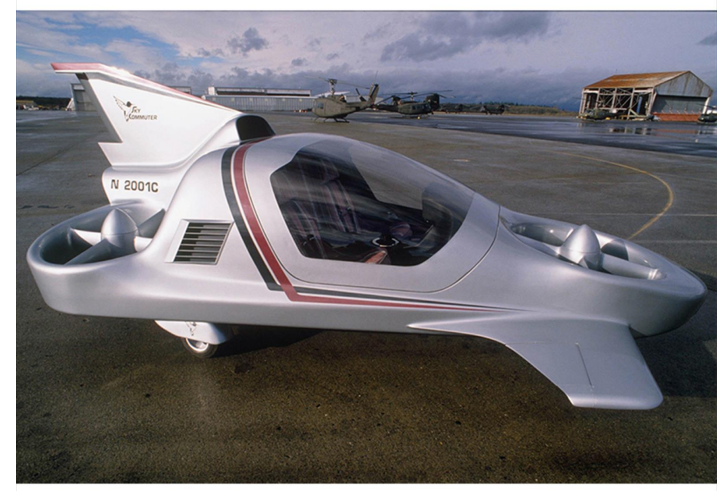

Fig. 9: The Sky Commuter in the 1980's. (https://www.arrajol.com/sites/default/files/2015/07/27/ 3-GAZ_da6aa2ce0ca341379d5f91a18013e38d.jpg)

pressure and needs arising from the rapid urbanization process and the economic growth across the world. Therefore, so far, the current technical trend of developing flying cars is to provide safe, green, fast and convenient human/freight transportation services in urban areas by using electric power, VTOL operation mode, and autonomous piloting techniques.

Now, some new start-up technology companies, ride-hailing companies (e.g., Uber), and some famous automobile and aircraft manufacturing companies (like Audi, Geely, Tesla, Toyota, Hyundai, Airbus, and Boeing) have engaged in flying 


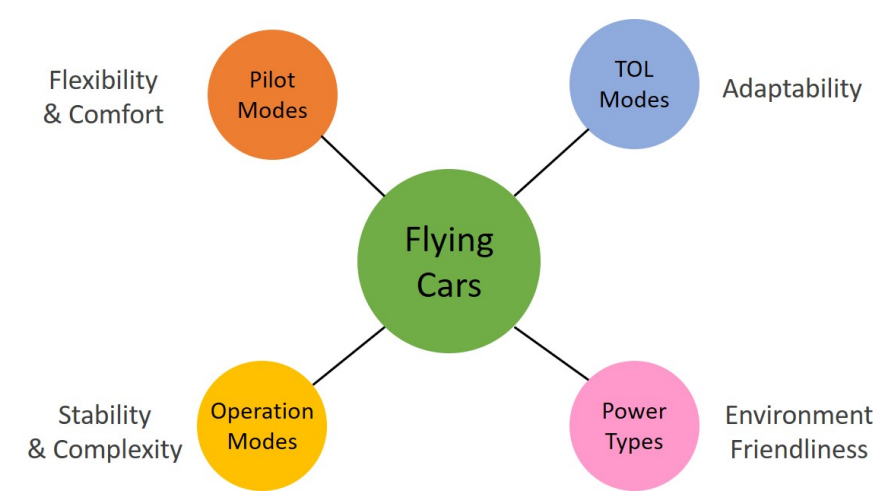

Fig. 10: The design aspects of flying cars.

cars area, and various types of flying cars have been designed, manufactured, and tested [11]- [31], which will be discussed in detail in the following sections ${ }^{2}$, making the commercialization of flying cars be on the near horizon soon. For example, Uber is working with its Elevate Network partners to launch fleets of small, electric VTOL aircraft in Dallas, Los Angeles, and Melbourne [12], and Morgan Stanley research says that "accelerating tech advances and investment could create a $\$ 1.5$ trillion market by 2040" [32].

\section{The Design of Flying Cars}

In this section, as presented in Fig. 10, we will respectively discuss the design of flying cars from the following four aspects: Take-off $\&$ landing (TOL) modes, pilot modes, operation modes, and power types, which are respectively related to the adaptability, flexibility \& comfort, stability \& complexity, environment friendliness of flying cars.

\section{A. TOL Modes}

Generally, there are four kinds of TOL modes for aerial vehicles:

1) VTOL Mode: The aerial vehicles can take off and land vertically. Under this mode, no runways are needed for both take-off and landing processes, resulting in more choices for TOL sites. In other words, the aerial vehicles with VTOL can be freer to reach anywhere as passengers want.

2) VTHL (Vertical Take-Off Horizontal Landing) Mode: The aerial vehicles take off vertically and return to the ground by taxiing along the runway. Then, runways are demanded in the landing process, while it is freer for the take-off process.

3) HTVL (Horizontal Take-Off Vertical Landing) Mode: The aerial vehicles taxi along the runway for a while before take-off, and land vertically to the destination. Thus, runways are required for the take-off process and it is flexible for the landing process.

4) HTOL (Horizontal TOL) Mode: The aerial vehicles taxi along the runway in both take-off and landing process. This mode is the most common one for fixed-wing aircraft, and

\footnotetext{
${ }^{2}$ In this article, the photos of the main modern designs of flying cars will not be presented, due to the page limitation, and please refer to the links [11] [31] to achieve if interested.
}

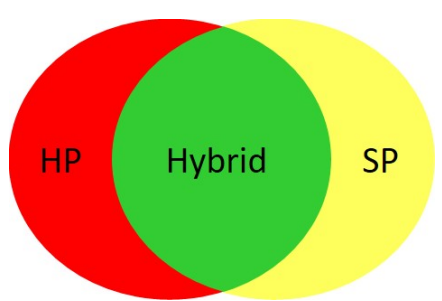

Fig. 11: Pilot modes of flying cars.

clearly, runways or ground supports are necessary, leading to high requirements for TOL sites.

Table II presents the comparisons among the four aforementioned TOL modes. It is easy to conclude that VTOL mode is the best choice for the flying cars operating in urban districts, in which runways are normally not available. The HTOL mode is a good direction for developing the flying cars for rural areas, in which there are enough spaces to build runways.

On the other hand, rotary wings or vertical fans should be equipped on the flying car to realize VTOL, while fixed/foldable wings are required for HTOL. From a technical viewpoint, compared with fixed/fold-able wings, rotary wings or vertical fans are more complex and expensive. So which type of TOL modes is the best one for the flying car depends on the application scenarios.

Table III presents the comparisons of the TOL modes among the main developing fly cars. Obviously, VTOL is the most popular one, as there are 20 kinds of fly cars adopting VTOL and only 5 kinds of them choose HTOL. The main reason for this observation is that most of these developing fly cars aim at the transportation market in an urban area in which there are few spaces for TOL operations. Moreover, as suggested in Table III rotary wings are necessary to realize VTOL. Then, compared with the flying cares with HTOL, the technical complexity and cost of the ones with VTOL will unavoidably increase. Consequently, a commercially viable solution is required to promote flying cares with VTOL into industrialization and commercialization.

On the contrary, as mentioned before, flying cars with HTOL is a suitable and commercial choice for rural areas or scenic spots, in which there is enough space to build runways for taxiing.

\section{B. Pilot Modes}

Pilot modes refer to how the operations of flying cars are under control during TOL and flying process, which is the most important role relating to the hardware cost, operating cost (e.g., fuel consumption and piloting cost), and safety of flying cars. Normally, there are three kinds of control modes for flying cars: Human-piloted (HP), self-piloted (SP) and hybrid modes, the relationship of which is depicted in Fig. 11. In the following, the three kinds of control modes will be respectively discussed.

1) HP Mode: Under this mode, fly cars are operated by pilots, like the traditional air-crafts, and then, driver license and/or pilot certificate are required. Thus, it will increase the labor cost (like the training fees and wages paid to the pilots), 
TABLE II: Comparisons Among The Four TOL Modes

\begin{tabular}{|c|c|c|c|c|c|}
\hline \hline Types & Technical Complexity & Runways/Supports & Maintenance Cost & Rotary wings/Vertical fans & Fixed wing \\
\hline VTOL & High & Not Required & High & Yes & No \\
\hline VTHL & Medium & Required & Medium & Yes & Yes \\
\hline HTVL & Medium & Required & Medium & Yes & Yes \\
\hline HTOL & Low & Required & Low & No & No \\
\hline \hline
\end{tabular}

TABLE III: TOL Modes of The Main Modern Flying Cars

\begin{tabular}{|c|c|c|c|c|c|}
\hline Flying Car & Company & TOL & Rotary wings/Fans & Propeller & Fixed/Foldable Wing \\
\hline PAL-V Liberty [11] & PAL-V & HTOL & Yes & Yes & No \\
\hline Elevate [12] & Uber & VTOL & Yes & No & No \\
\hline Heaviside [13] & Kitty Hawk & HTOL & No & Yes & Yes \\
\hline Flyer [13] & Kitty Hawk & VTOL & Yes & No & No \\
\hline Vahana [14] & Airbus & VTOL & Yes & No & No \\
\hline AeroMobil V. 4.0 [15] & AeroMobil & HTOL & No & No & Yes \\
\hline AeroMobil V. 5.0 [15] & AeroMobil & VTOL & Yes & No & No \\
\hline VoloCity [16] & Volocopter & VTOL & Yes & No & No \\
\hline MOOG [17] & SureFly & VTOL & Yes & No & No \\
\hline BlackFly [18] & Opener & VTOL & Yes & No & No \\
\hline The Transition [19] & Terrafugia & HTOL & No & Yes & Yes \\
\hline The TF-2 [19] & Terrafugia & VTOL & Yes & No & No \\
\hline EHang AAV [20] & EHang & VTOL & Yes & No & No \\
\hline Cora $[21]$ & Wisk & VTOL & Yes & Yes & Yes \\
\hline Joy Aviation Air Taxi [22] & Joby Aviation & VTOL & Yes & No & No \\
\hline Jaunt Aircraft [23] & Jaunt Air Mobility & VTOL & Yes & Yes & No \\
\hline Passenger Air Vehicle [24] & Boeing NeXt & VTOL & Yes & Yes & Yes \\
\hline Cargo Air Vehicle [24] & Boeing NeXt & VTOL & Yes & No & No \\
\hline SD-XX [25] & SkyDrive & VTOL & Yes & No & No \\
\hline The Volante Vision Concept [26] & Aston Martin & VTOL & Yes & No & No \\
\hline Moller M400 Skycar [27] & Moller International & VTOL & Yes & No & No \\
\hline The Nexus [28] & Bell & HTOL & Yes & No & No \\
\hline WD-1 [29] & Detroit Flying Cars & VTOL & Yes & Yes & Yes \\
\hline DR-7 [30] & Delorean Aerospace & VTOL & Yes & No & Yes \\
\hline The Pop.Up Next [31] & Audi & VTOL & Yes & No & Yes \\
\hline
\end{tabular}

leading to a considerable burden to the commercial operation of flying cars. Because the ratio of driver/pilot to passengers per fly car is much lower compared with traditional public air-crafts. However, high operation freedom to flexibly react to some emergence cases can be realized under the humanpilot mode, so as to enhance the robustness of flying cars.

2) SP Mode: Under SP mode, flying cars are permitted to perform all operations in a way fully autonomous with no need for pilots, showing reduced operating costs compared with HP mode. Currently, SP mode is a main and popular choice for the developing flying cars, as shown in Table IV in which SP mode has been supported by 13 out 25 kinds of the developing flying cars (including the ones with hybrid mode), benefiting from the maturity of autonomous control and navigation technologies that makes the vehicle smarter and safer. In the future, it will be more reliable and efficient by exploiting the development of artificial intelligence technologies. For the commercial applications of flying cars, SP mode is especially fit for the scenarios with public air shuttles with fixed routing.

3) Hybrid Mode: In order to fully make use of the advantages of the two aforementioned control modes, the hybrid mode has been proposed and adopted by some of the developing flying cars. Though the manufacturing cost of the flying care with hybrid mode will increase compared with two other modes, hybrid mode is still a good candidate for some applications, like flexible transportation without fixed routing lines. As a practical example, flying cars with hybrid mode will work well for tours and sightseeing spots.

\section{Operation Modes}

As there exist various kinds of TOL modes and types of wings, flying cars are endowed with different operation modes, such as airplane, helicopter, and hybrid modes.

1) Airplane mode: There is one kind of flying cars working like traditional airplanes, Heaviside produced by Kitty Hawk [13] and the Nexus by Bell [28]. In other words, Heaviside only can take-off, fly, and land like the traditional airplane, but not run on the roads like cars. Therefore, considerable space is required for the TOL behaviors of this kind of flying car, making it unsuitable to work in urban areas.

2) Helicopter Mode: Helicopter mode is a popular operation mode for flying cars, which fully inherits the TOL and flying characteristics of helicopters. Thanks to the unique TOL behaviors of helicopter mode that can save a lot of space, it is straightforward to see that the flying cars with this mode are quite suitable for transportation in urban areas. As suggested in Table IV, there are 10 kind of flying cars adopting helicopter mode, i.e., Flyer by Kitty Hawk [13], Volocity by Volocopter [16], MOOG by SureFly [17], EHang AAV by EHang [20], 
TABLE IV: Comparisons Among The Main Modern Flying Cars

\begin{tabular}{|c|c|c|c|c|c|c|}
\hline Flying Car & Speed $(\mathrm{km} / \mathrm{h})$ & Range $(\mathrm{km})$ & Operation Modes & Power Types & Control Modes & Passengers \\
\hline PAL-V Liberty [11] & $160^{\mathrm{T} 0}$ & $400^{\mathrm{T} 1}$ & Helicopter-car & Hydrocarbon & $\mathrm{HP}$ & Multiple \\
\hline Elevate [12] & $241.4 \sim 370.15^{\mathrm{T} 2}$ & $321.87^{\mathrm{T} 3}$ & Helicopter-airplane & Electric & HP & Multiple \\
\hline Heaviside [13] & - & - & Airplane & Electric & HP & Single \\
\hline Flyer [13] & - & - & Helicopter & Electric & HP & Single \\
\hline Vahana [14] & 190 & 50 & Helicopter-airplane & Electric & SP & Single \\
\hline AeroMobil V. 4.0 [15] & $360^{\mathrm{T} 0}$ & 750 & Airplane-car & Hybrid & $\mathrm{HP}$ & Multiple \\
\hline AeroMobil V. 5.0 [15] & - & - & Helicopter-car & Electric & Hybrid & Multiple \\
\hline VoloCity [16] & $80 \sim 100^{\mathrm{T} 4}$ & $30-35$ & Helicopter & Electric & Hybrid & Multiple \\
\hline MOOG [17] & 120.7 & - & Helicopter & Electric & Hybrid & Multiple \\
\hline BlackFly [18] & $>128.75^{\mathrm{T} 5}$ & $64.37^{\mathrm{T} 6}$ & Helicopter-airplane & Electric & HP & Single \\
\hline The Transition [19] & $161^{\mathrm{T} 0}$ & 644 & Airplane-car & Hydrocarbon $^{\mathrm{T} 7}$ & HP & Multiple \\
\hline The TF-2 [19] & $230^{\mathrm{T} 0}$ & 300 & Helicopter-airplane & Hydrocarbon $^{\mathrm{T} 8}$ & HP & Multiple \\
\hline EHang AAV [20] & $130^{\mathrm{T9}}$ & - & Helicopter & Electric & SP & Multiple \\
\hline Cora [21] & 160 & 40.23 & Helicopter-airplane & Electric & SP & Multiple \\
\hline Joy Aviation Air Taxi [22] & 321.87 & 241.4 & Helicopter-airplane & Electric & HP & Multiple \\
\hline Jaunt Aircraft [23] & $281.64^{\mathrm{T} 9}$ & - & Helicopter-airplane & Electric & $\mathrm{HP}$ & Multiple \\
\hline Passenger Air Vehicle [24] & - & - & Helicopter-airplane & Electric & Hybrid & Multiple \\
\hline Cargo Air Vehicle [24] & - & $\overline{-}$ & Helicopter & Electric & SP & None \\
\hline SD-XX [25] & 60 & $20-30$ & Helicopter & Electric & Hybrid & Multiple \\
\hline The Volante Vision Concept [26] & - & - & Helicopter & Hybrid & SP & Multiple \\
\hline Moller M400 Skycar [27] & 533 & 1213 & Helicopter & Electric & HP & Multiple \\
\hline The Nexus [28] & $241.4^{\mathrm{T} 0}$ & 241.4 & Helicopter & Hybrid & HP & Multiple \\
\hline WD-1 [29] & 201.17 & 643.74 & Helicopter-car & Hybrid & Hybrid & Multiple \\
\hline DR-7 [30] & 241 & 193 & Helicopter & Electric & SP & Multiple \\
\hline The Pop.Up Next [31] & - & - & Helicopter-car & Electric & SP & Multiple \\
\hline
\end{tabular}

T0: The maximum speed;

T1: The maximum range is $400 \mathrm{~km}$ and $500 \mathrm{~km}$ for two and a single person, respectively;

T2: A desirable speed for VTOL vehicle;

T3: Obtained at the best range flight speed;

T4: The optima cruise speed range with the trade-off between time efficiency, and cost, safe operation at low altitudes and low noise;

T5: The speed is no more than $99.78 \mathrm{~km} / \mathrm{h}$ for the USA;

T6: Obtained with the load $200 \mathrm{lbs}$, while the rang is $40.23 \mathrm{~km}$ for the USA with load $200 \mathrm{lbs}$;

T7: Hybrid-electric motors for drive mode;

T8: Under air flying mode, 8 electric motors are powered initially by a turbine generator and ultimately powered by batteries, while for drive mode it will be hybrid-electrically powered initially, and become purely electric as practical;

T9: The maximum cruise speed.

Cargo Air Vehicle by Boeing NeXt [24], SD-XX by SkyDrive [25], the Volante Vision Concept by Aston Martin [26], Moller M400 Skycar by Moller International [27], the Nexus by Bell [28], and DR-7 by Delorean Aerospace [30].

3) Hybrid Mode: In order to satisfy different application demands, the designers also present some other hybrid modes to jointly utilize the advantages of each operation mode.

a) Helicopter-car mode: Under mode, the flying cars can not only take-off, fly and land like helicopters, but also run on the road like traditional cars. Generally, foldable rotary wings are equipped with this kind of flying cars to achieve good performance while running on the roads. The helicoptercar mode is applied on PAL-V Liberty by PAL-V [11] and AeroMobil V. 5.0 by AeroMobil [15].

b) Helicopter-airplane mode: Under this mode, the flying cars take-off and land like helicopters and fly like airplanes. The main difference between Helicopter-airplane mode and Helicopter mode is that fixed/foldable wings and horizontal propellers are applied under helicopter-airplane mode to attain better flying performance. As helicopter-airplane mode shows high adaptability for urban areas, it is the most popular operation mode and there are 8 kinds of flying cars proposed/designed by the manufacturers, as shown in Table IV.

c) Airplane-car mode: Foldable wings are developed for this kind of flying cares to achieve both good flying performance in the air and running performance on the road. Then, the flying cars should be taxied for a while on the road to takeoff and land. Due to the rigid requirement on the TOL space, the flying cars under airplane-car mode are not suitable for urban areas, in which normally there is no enough space for building runways, and maybe a good choice for rural areas or some specific scenarios, like large-scale outdoor scenic sites. As shown in Table IV, there are only two types of flying cars adopted airplane-car mode: AeroMobil V. 4.0 by AeroMobil [15], the Transition by Terrafugia [19], WD-1 by Detroit Flying Cars [29], and the Pop.Up Next by Audi [31].

\section{Power Types}

Duo to various application purposes, there are mainly three power types for flying cars: electric power, hydrocarbon fuel, and hybrid power.

1) Electric Power: Electric propulsion has already been chosen as the preferable propulsion for flying cars as it exhibits many desirable characteristics acquiring low emissions and low noise pollution. As electric batteries are the obvious energy source for the flying cars serving in urban areas (18 out of 25 kinds of flying cars are with electric power, as suggested in Table IV), there are some specific requirements 


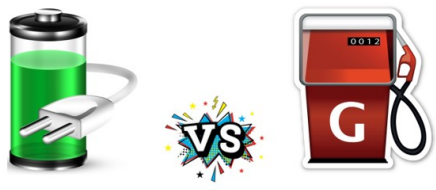

\section{Performance}

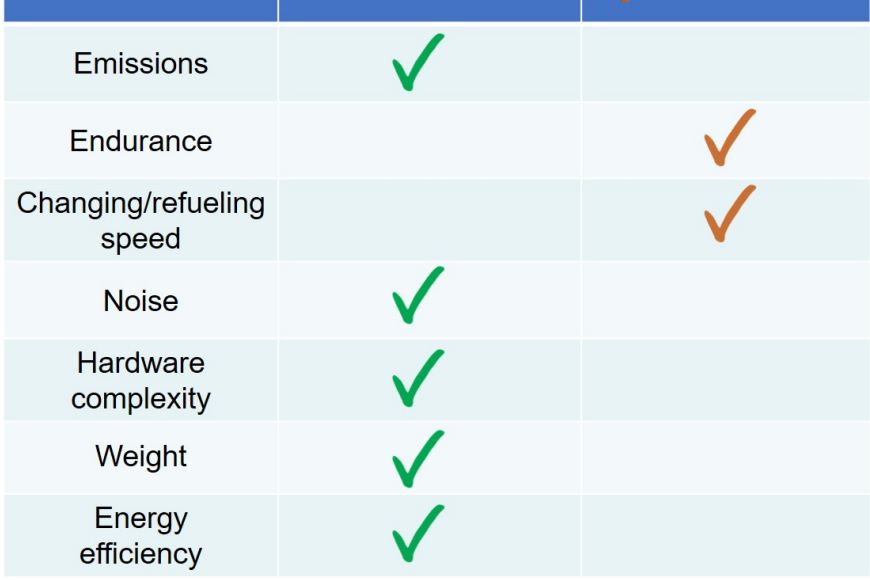

Fig. 12: Comparisons between electric power and hydrocarbon fuel.

for the batteries, e.g., the energy density (the amount of energy per unit weight provided by the batteries), the recharging time (how much time is needed for the battery to be back to a nearly full charge), the cycle life (the number of complete discharge/charge cycles the battery can support before the capacity falls under $80 \%$ of its original capacity), and the average price of the battery (the cost per kilowatt-hour), which are important to support the demands of the highfrequency ride-sharing operations of flying cars in urban public transportation. Moreover, there is another important issue that should be highlighted and treated: the pollution arisen from the heavy metals and acids of the wasted batteries. Thus, green battery technologies with high energy density, large cycle life, and low cost are urgent to develop for the incoming flying cars services.

2) Hydrocarbon Fuel: Some designers try to compromise the merits of both traditional cars and air-crafts to enable flying cars to run on the ground like traditional automobiles and to fly in the air like airplanes/helicopters, e.g., PAL-V Liberty by PAL-V [11], and the Transition and TF-2 by Terrafugia [19]. Thus, compared with the flying cars with electric power, it is obvious that there exists a drawback with the flying car with hydrocarbon fuel: noise and air pollution incurred by an internal-combustion engine, though the hardware cost and manufacturing complexity of the flying car can be highly saved compared the flying cars with hybrid power which will be discussed in next part.

3) Hybrid Power: As shown by the comparisons in Fig. 12 , electric power outperforms hydrocarbon fuel in terms of emissions, noise, hardware complexity, weight, and energy efficiency, which shows electric power is more suitable for flying cars, especially for urban areas. Furthermore, one can also see that the endurance and refueling speed of hydrocarbon fuel are better than electric power, making it suitable for longdistance transportation designs. Obviously, the charging speed problem of electric power can be simply solved by replacing batteries at TOL sites other than directly charging the batteries.

In order to combine the advantages of both electric power and hydrocarbon fuel, a hybrid power option is proposed realized for flying cars, which is similar to what happens in the automobile industry. However, such kind of design will inevitably increase the hardware complexity and cost of flying cars, and further will result in poor economic competitiveness.

From the existing designs of flying cars listed in Table IV, hybrid power is a good candidate power configuration to realize the hybrid transportation functions on the ground and in the car, which has been utilized by AeroMobil V. 4.0 by AeroMobil [15], SD-XX by SkyDrive [25], the Nexus by Bell [28], and WD-1 by Detroit Flying Cars [29]. For example, with hybrid power configuration, AeroMobil V. 4.0 by AeroMobil on the ground under drive mode is powered by a hybridelectric system, and the same engine has been employed that powers the vehicle in the air under flight mode, which in turn powers a pair of electric motors located in the front axle [15].

Therefore, we can conclude that electric power is the greenest and cost-saving power configuration for flying cars, and hybrid power is a reasonable and commercial choice to facilitate hybrid transportation functions on flying cars.

\section{The Design of FCTS}

In the previous section, the issues related to the design of flying cars have been discussed. In this section, we will introduce FCTS from the following three aspects: Path and trajectory planning, supporting facilities and commercial designs, which are the key problems in large-scale operations of FCTS.

\section{A. Path/Trajectory Planning}

Path/trajectory planning is a crucial issue in the field of FCTS. Indeed, the main trend for flying cars is to operate over the urban areas, in order to achieve shorter transportation times and relieve road congestion. The high operating speed in the air may cause chaos and collisions among the flying cars and buildings, then the extremely accurate and scientific path and trajectory planning are required from the specific department of the government. However, to this day, there is no provision yet for FCTS, especially for dedicated VTOL paths/routes. Therefore, particular care should be paid in generating flying path/trajectory that could be executed in the air above the city, but simultaneously efficient and safe for the transportation, in terms of avoiding any unnecessary flying and possible security vulnerabilities. The content architecture of this subsection is given in Fig. 13.

1) Position \& Navigation: During the flight process, the most important thing to promise the flying car following the pre-designed path is the accurate position and navigation. Therefore, attention should be focused on how to achieve precise information on position and navigation.

So far, there are various methods developed to obtain the two-dimensional position information of the object, 


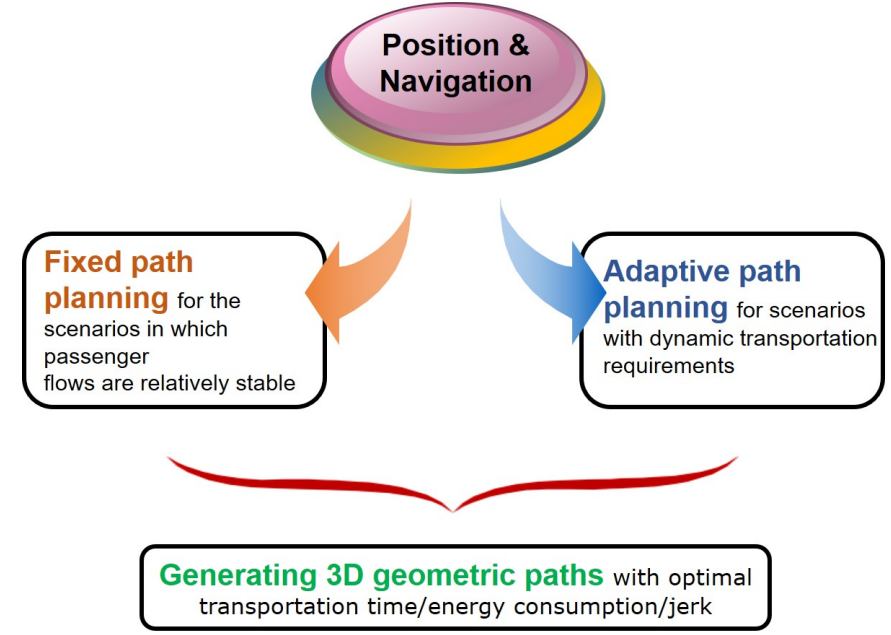

Fig. 13: Path planning for FCTS.

such as Global Positioning System (GPS), and cellular network/wireless fidelity (Wi-Fi). These technologies have been testified and well applied to numerous ground scenarios in our common life. However, as the flying cars normally aim at operating on the height ranging from tens to hundreds of meters, accurate three-dimensional position information is required for FCTS to precisely determine where the flying car is and which the height the flying car is on.

Intuitionally, radars are an effective tool to work out this problem for the ground control centers. On the contrary, on the flying car's side, it is still a challenge but can be relieved by new position and navigation algorithms adopting some reference nodes, e.g., the base stations on the roof of the buildings/hills and the low attitude platforms serving as aerial access points for the 5th or future generation of wireless communications.

2) The Objects of Path/Trajectory Planning: The purpose of the path/trajectory planning for FCTS is to generate 3D geometric paths, from an initial to a final point, passing through pre-defined via-points, either in the restricted spaces identified by the government or in the conservation spaces of the wildlife.

The algorithms for path/trajectory planning are usually named by the functions that are optimized in terms of transportation time/energy consumption/jerk (i.e., the derivative of the acceleration, which related to the passenger experience), which are devoted to satisfying the requirements in the application scenarios of flying cars.

Moreover, the major difference between the path/trajectory planning for FCTS and the one for the traditional ground public transportation system is the passenger experience while traveling in the air that may also be affected by the weather conditions along the path/trajectory.

3) Fixed Path Planning: For public transportation in urban areas in which passenger flows are relatively stable, it is commercially to provide fixed paths for flying cars, which likes the ground public bus transportation system. Therefore, there are some similar design methods and key problems (like traffic load, etc.) during the fixed path planing with the traditional bus transportation system, but some unique features of FCTS should be carefully treated. For example, the safety of TOL behaviors, the noise pollution generated during the TOL process, and the forbidden spaces announced by the government must be taken into account, while choosing the TOL sites for flying cars. Moreover, fixed paths should be dynamically maintained and updated according to the developments of the city and the regulations presented by the government.

4) Adaptive Path Planning: Other than the public transportation discussed in the last part, there are also some other application scenarios for flying cars in which transportation requirements are dynamic, like short-distance travel and sightseeing, and customized personalized services. Therefore, the operators of flying cars should be able to quickly offer the customers convenient and efficient path planning services according to their requirements.

\section{B. Support Facilities}

Similar to traditional ground traffic systems, some support facilities are essential to set up and maintain efficient services by flying cars. Generally, in view of the functions, the support facilities of FCTS can be divided into the following three kinds: communication systems, TOL sites, and storage and maintenance facilities (SMF), as presented in Fig. 14.

1) Communication Systems: Communication systems are pretty crucial for FCTS, which serve as the role of the central nervous system to collect, deliver, switch and broadcast information among flying cars, control centers, and information service providers. In more detail, there are two kinds of communication systems that are needed to respectively supply FCTS and the passengers with control information and data communication services, as discussed in the following.

a) Control signal system: The aim of the control signal system for FCTS is to ensure the efficient organization of the flying transportation, allowing flying cars from all participating companies to provided high-quality service, as well to profit from the service. Via the path planning management, TOL sites can be served by flying cars in the event of accidents or path obstructions, or path re-planning service is available for the flying cars facing the case of the cancellation/unavailability of the previously defined path. Additionally, flying cars can also be sent into service rapidly to avoid overcrowding, or even to breakdowns. The monitoring data collected through the control system can also be used to improve timetables and optimize flying car usage.

The key goals of the control signal system for FCTS can be summarized as i) the efficient and intelligent management of public/personal transportation; ii) the optimization of traffic flows leading to better passenger experience and reductions in energy consumption; iii) the integration with the other public transportation systems; iv) the promotion of the utilization of FCTS by making it more attractive.

b) Data communication system: The purpose of the data communication system for FCTS is to provide normal voice and multimedia data communications for the passengers, which is an essential part of FCTS to enhance the passenger 


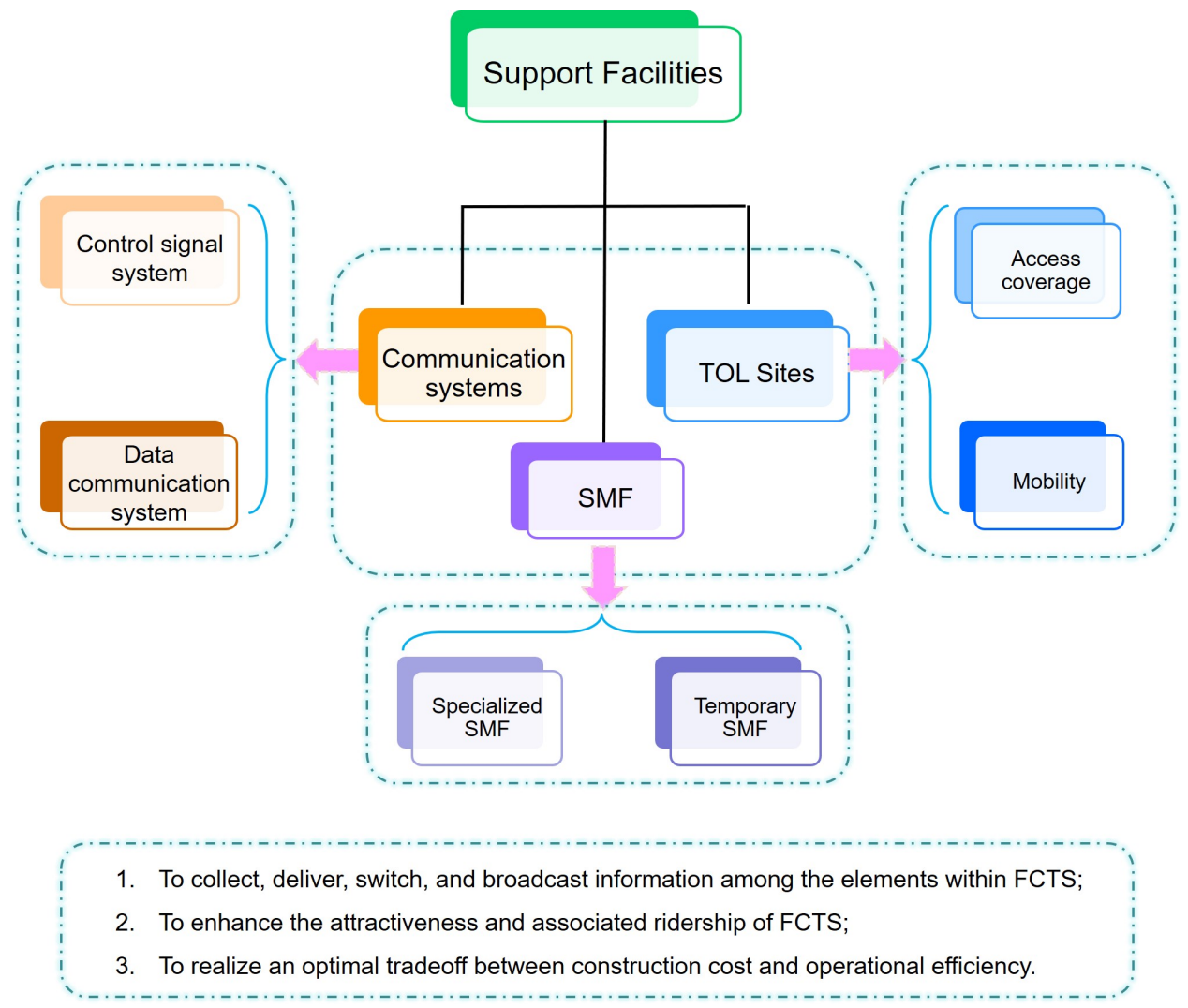

Fig. 14: Support facilities for FCTS.

experience during the transportation process. The key requirement of the data communication system for FCTS can be concluded as reliable, seamless, robust, and high-speed data transmissions.

Observing the fact that flying cars are planned to bring to the altitude of around hundreds of meters above ground, the coverage performance of traditional terrestrial wireless communication systems in the operating space of flying cars may not be as well as the one on the ground. Therefore, a new communication network design and planning must be conducted. For example, the developing high/low altitude platforms and LEO satellite communication systems for $5 \mathrm{G}$ and future generation of wireless communications, which aim at providing seamless connections across the world, can be exploited to build up the data communication system for FCTS.

2) TOL Sites: As FCTS is able to provide direct point-topoint transportation services, the placements and constructions of TOL sites should enhance the attractiveness and associated ridership of FCTS via providing high-quality services and experience to guarantee efficient mobility for all riders within the service area, especially in urban areas.

Differing from traditional public transportation systems, like public road traffic systems (bus and train) in the cities, the transportability of the flying cars in FCTS cannot be as large as the ones supplied by buses or trains. Thus, the commercial positioning of FCTS should be to offer fast and convenient transportation services to premium passengers who are in need and can afford the transportation fees, at least in the initial phase, and then the survival of FCTS can be promised in the commercialization-orientated market. Therefore, in urban areas, the locations of TOL sites should be in the business zones (e.g., central business districts), the connecting points to other transportation systems (e.g., bus stops, subway/train stations, airports, etc.), and some other functional places (e.g., hospitals, libraries, schools, etc.)

Specifically, the problem of determining TOL site placements in special areas (e.g., business zones) is not trivial as a balance between access coverage and mobility must be achieved in general. Hence, these two factors should be taken into consideration simultaneously and methodologies for the determination and optimization of TOL site placements should be proposed at the network level.

Apparently, the density and locations of TOL sites usually determine the access coverage of the FCTS service, which is similar to the traditional bus transportation system. Some existing researches revealed that access/egress distance is negatively and significantly related to transit patronage. Consequently, the spacing of TOL sites should suffer an upper threshold so as to guarantee that passengers complete their access/egress trips in a reasonable and acceptable amount of time. Generally, such a maximum spacing threshold is location-specific, depending on many local considerations (e.g., service regions, road hierarchies, local demands).

Apart from access coverage, mobility is the other prominent factor greatly affecting the system performance and level of service of FCTS. Then, efficient mobility is preferred, which closely related to TOL site placements. Due to the fact that 
fewer TOL sites generate less dwell time at TOL sites and subsequently contribute to higher utilization of the fly cars and lower construction costs of the whole FCTS, minimizing the number of TOL sites is supposed to be one of the key principles of TOL site deployments.

Therefore, these two mutually exclusive factors must be optimally trade-offed at the whole FCTS level during the process of determining TOL sites, while the location specifics in the given areas.

3) SMF: Though no roads or tracks are needed in FCTS, some necessary supporting facilities are required to run FCTS as existing traditional traffic systems do [33], such as SMF, which will be discussed in this part.

Roughly speaking, there are two kinds of SMF according to the unique properties of FCTS and the functions of facilities: 1) Specialized SMF and 2) Temporary SMF. The first one is specialized to provide daily storage and maintenance for flying cars, which should be large enough to satisfy all fly cars in their service zones, and the second one is designed and integrated with normal TOL sites to supply temporary storage and maintenance services to the flying cars that are out of order.

Some important rules for the design and construction of specialized SMF must be satisfied. First, SMF should be free of environmental contamination (including noise/light/heavy metals pollution). Second, the size of SMF should be large enough to accommodate all present requirements and any potential future expansion. Third, SMF should be able to offer comprehensive functions, e.g., general maintenance and storage, fare service, fueling, exterior washing, and interior cleaning. The last, additional space will be provided for staff offices, training/diner/locker rooms, associated toilet facilities, on-site parking for employees/visitors.

Observing these requirements, intuitively, it is reasonable for SMF to be constructed in suburb areas which also should be far from living communities, so as to adapt the constraint factors including availability and affordability of suitable land and air spaces, real estate, and redevelopment interests, zoning regulations. Therefore, the optimization of the placement of SMF is a comprehensive problem that should deal with the relationship between construction cost and operational efficiency.

For temporary SMF, the design and construction are not as complicated as the ones of specialized SMF, as temporary storage and maintenance functions are easily integrated into normal TOL sites. However, it will unavoidably increase the construction and operation costs of TOL sites which normally locate in urban areas, leading to the necessity of implementing the trade-off between commercially operation cost and supporting capacity.

\section{Commercial Designs}

As a novel transportation method, which has not yet been put into commercial operation, commercial designs of FCTS should be carefully implemented. In other words, commercial operation modes are required to be proposed and designed to promise the viability of FCTS in the real market. In the following, we will describe the commercial designs of FCTS from two aspects: Market opportunities and commercial models. The relationship between the main contents is depicted in Fig. 15.

1) Market Opportunities: The main market opportunities arise from the ability of FCTS to relieve the pressure of the increasing congestion and overcrowding in cities, which are caused by rapid urban development and increase in urban population. Specifically, FCTS is capable of satisfying the main requirements of the urban mobility or transportation in cities, which can roughly be categorized into two kinds: Human (public and individual), and freight transportation, while taking the pressure off roads and other infrastructure and reducing transportation costs.

a) Human transportation: Now, human transportation is popularly regarded as the first design function of FCTS to move people in a faster way in congested cities. Because the NGS is first explored for daily large-scale utilization, leading to considerable spatial gain for transportation systems. Therefore, as discussed in previous sections, profiting from the intersection of many technologies, such as ultra-efficient batteries, autonomous systems, communication technologies, and advanced manufacturing processes, FCTS can ensure a faster, cheaper, cleaner, and safer transport solution for both public and individual transportation activities, compared to traditional terrestrial transportation systems.

b) Freight transportation: Obviously, similar to human transportation, FCTS is also able to offer door-to-door freight transport services, though a load of flying cars cannot as large as the trucks or trains running on the ground. For example, some kinds of goods with high priority but lightweight and limited size can be delivered via flying cars. On the other hand, the load of flying cars is much larger than the one of normal developing commercial freight UAV, e.g., drones for faster delivery developed by JD.com [34] and Amazon [35], which is used to deliver packets. Therefore, freight flying cars are a promising solution to fill the market blank in the aspects of both the load and delivery range between the traditional on-ground freight transportation systems and the developing freight drones.

2) Commercial Models: In the previous part, the commercial application opportunities of FCTS have been discussed. In this part, the commercial models of FCTS will be introduced to facilitate the readers to understand how to implement FCTS in the real market. In general, so far, there are two potential types of commercial models for FCTS according to commercial operation behaviors, which will be respectively demonstrated in the following.

a) Public transportation mode: Under this mode, serving as an alternative solution to traffic congestion issues that plague the cities, FCTS is applied to play a similar role with traditional transportation systems, like public bus/train/subway systems, to transport passengers or goods with fixed routines according to predesigned timetables. Then, FCTS here will be part of the public transportation system in a city or an area, which should apply for special operation grants from local government. Moreover, it must be jointly designed and optimized to collaborate with other public transportation systems 


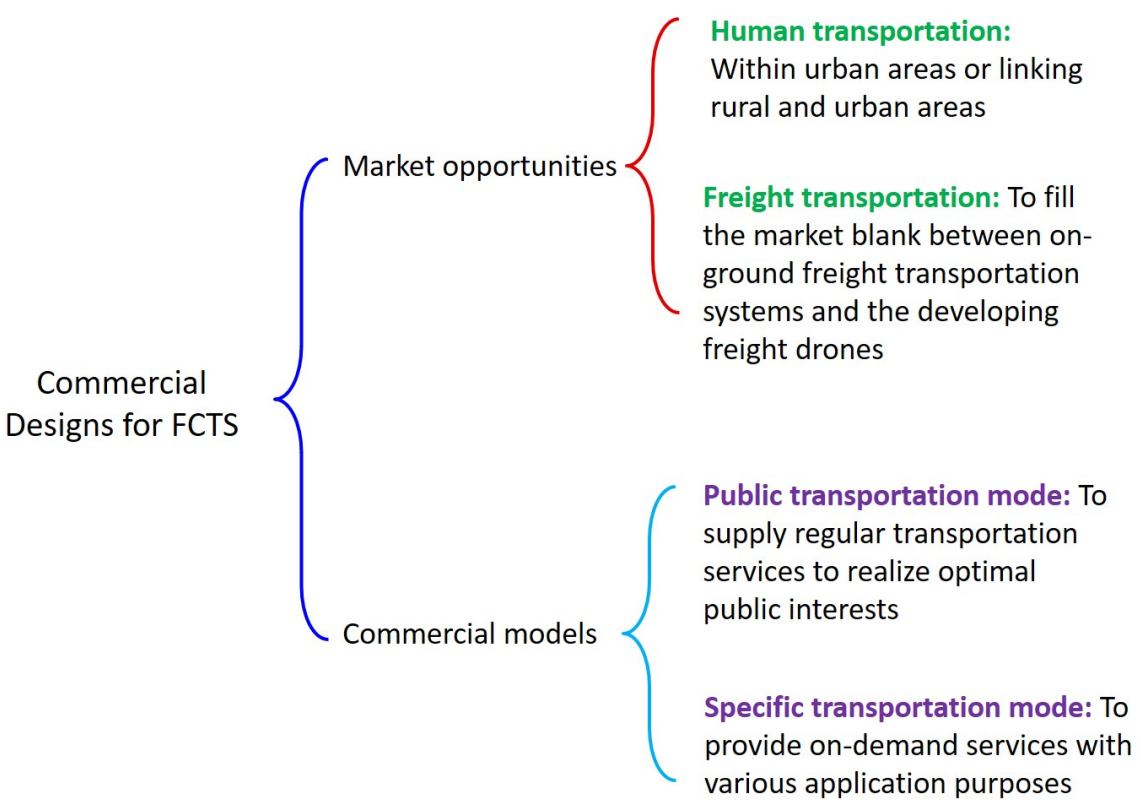

Fig. 15: Commercial designs for FCTS.

to realize optimal public interests. Therefore, it is obvious that the construction and operation of the FCTS under this mode are a complicate and challenge task, influenced by various kind of factors include local political agendas, real estate, and redevelopment interests, city regulation, development and planning, availability of suitable land, conflicts with traditional transportation systems, etc.

b) Specific transportation mode: Benefiting from its unique characteristics, FCTS has been designed for some specific application scenarios for tourism, patrol, monitoring, inspection, anomaly detection/prevention, search and rescue purposes, such as sightseeing in scenic spots, mine/dam inspection, traffic/environmental monitoring, border/harbor patrol, police surveillance, aerial photography, imaging, and mapping, etc. Under this mode, the design and operation of FCTS under these on-demand application modes are more flexible and convenient, compared with the ones of FCTS for public transportation applications.

\section{The Challenges of FCTS}

Despite several flying car prototypes have already proposed, manufactured and testified [11]- [31], putting FCTS into largescale practical commercial applications still has a long way to go, as there exist considerable challenges not only from technical aspects but also from commercial and ethical aspects [36], which will be illustrated and discussed in detail as follows. The architecture of the main contents in this section is presented in Fig. 16.

\section{A. Safety Issues}

The most important challenge faced by the commercial operations of FCTS is the safety issues arising from various facets, such as flying car level safety (including passenger protection and robustness to weather conditions), systemlevel safety (including collision/obstacle avoidance, search and rescue, intrusion and attack in control networks), and environmental safety.

1) Passenger Protection: Differing from the traditional terrestrial transportation systems, flying cars normally operate on the height from tens of meters to hundreds of meters to transfer people from one place to another. Then, how to avoid the following potential cases happen and how to protect the passengers? For instance, how to deal with the case that a flying car malfunctions (e.g., at risk of short circuit or fire) over a busy city and falls down on a crowd of people? Specific survival equipment and emergency mechanism must be invented, developed and equipped with flying cars to promise the safety of the passengers, as well as to guarantee the safety of the people and properties on the ground. These necessary requirements are the first and the most important rule for FCTS that must be satisfied and obeyed, though it unavoidably poses numerous engineering challenges to the designers and manufacturers of flying cars.

2) Robustness for Weather Conditions: As aforementioned, flying cars work in the NGS, leading to the sensitivity of the weather conditions in the operating space. Thus, the robustness of flying cars towards to some extreme weather conditions, e.g., flash, heavy wind and rain brought by severe convective weather, must be taken into consideration during the design stage, though weather conditions can be forecast and then reported to the operator/pilot of flying cars.

3) Collision/Obstacle Avoidance: As there are some other flying vehicles sharing the air space with flying cars, e.g., UAV, helicopters, etc., collisions may happen among flying cars and between flying cars and some other flying vehicles. Moreover, normally, the threat of collisions also comes from the obstacles in the NGS constraining power-lines, skyscrapers in the cities, mountains, etc. Therefore, similar to traditional 


\section{The Challenges of FCTS}

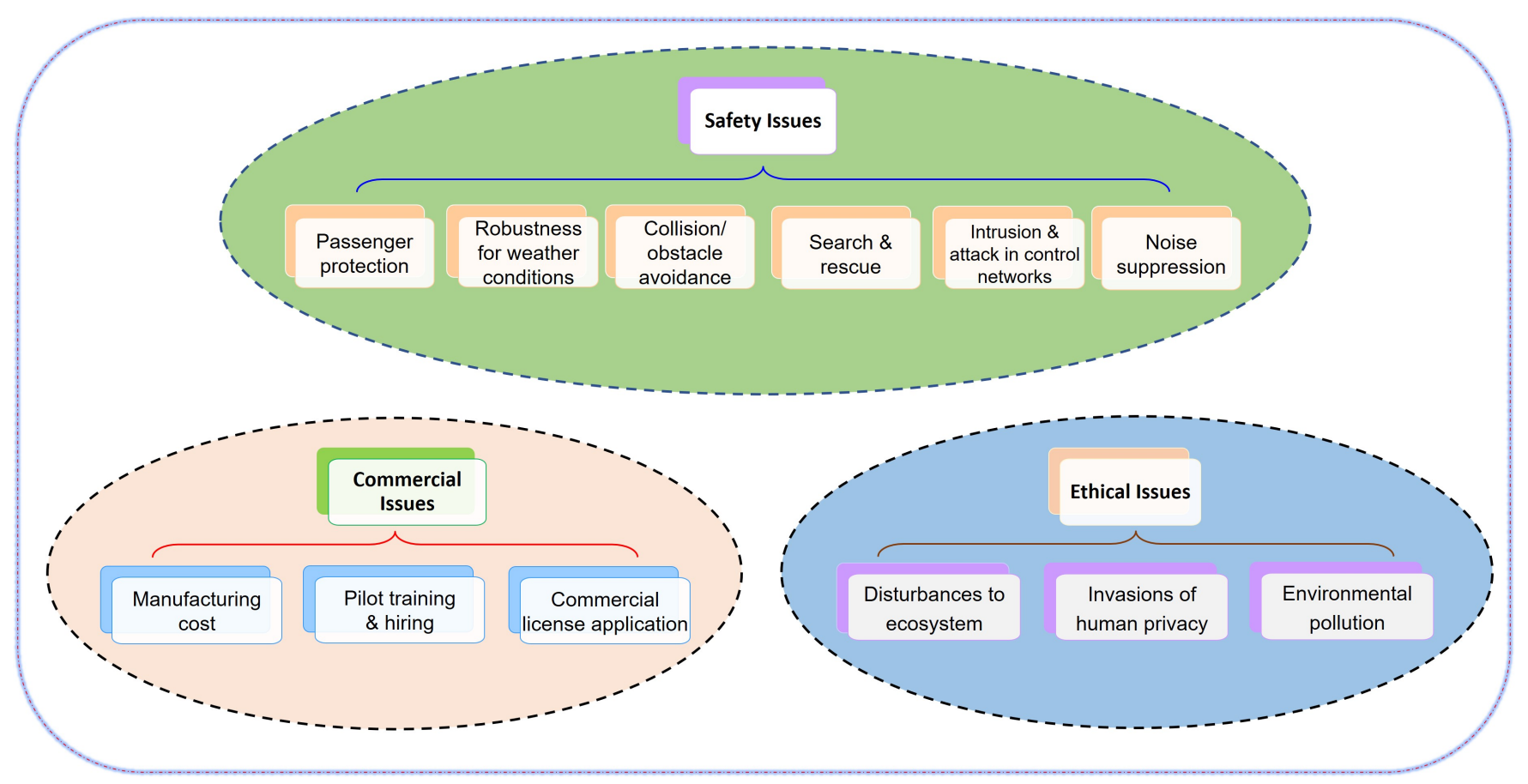

Fig. 16: The Challenges of FCTS.

air-crafts (helicopters and airplanes), devices for early warning and detecting should be equipped with flying cars to have the ability of obstacle avoidance. On the other hand, auxiliary ground surveillance network is suggested to be built up to monitor the main operating space of FCTS, like in the urban areas, to provide on-time traffic control and routine schedule services.

4) Search \& Rescue: In emergency cases of crashes, FCTS should be able to quickly respond and to start an emergency treatment plan. Specifically, search and rescue operations are required to carried out to help the passengers and the crashed vehicle, once alarms arise. Then, some resources including humans and materials must be optimally prepared, allocated and distributed in advance in the serving areas of FCTS. Obviously, the related aspects of search and rescue are parts of the system-level design and construction of FCTS.

5) Intrusion \& Attack in Control Networks: In the information era, it is impossible to isolate FCTS away from information networks, resulting in threatens of the control network of FCTS being intruded and attacked. In order to solve this fatal menace, some similar protection schemes adopted in some other transportation systems can also be referred to and implemented in FCTS to safeguard the brain of FCTS to avoid flying cars being hijacked and manipulated.

6) Noise Suppression: Since flying cars normally aim at operating in urban environments that have already been tightly regulated on noise constraint, it is a challenge to suppress the noise coming from flying and TOL behaviors. Especially, when flying cars are put into large-scale commercial operations, it is difficult for flying cars, which may carry out hundreds of TOL every hour in urban areas, to be exceptionally quiet. In order to suppress noise, some methods can be worked out not only from the flying car level but also form the CTS level, e.g., low noise propellers/(electric) motors can be developed and improved for flying cars, and the noise index considered in the deployments and designs of TOL sites.

\section{B. Commercial Issues}

In this subsection, we will discuss the commercial issues suffered by FCTS in large-scale commercial applications.

1) Manufacturing Cost: The first practical challenge that hinders FCTS from being commercialized is the manufacturing cost of flying cars. Thinking about the case of the largescale commercial operation of FCTS, likely, hundreds of, even thousands of sustainable and cost-efficient flying cares are needed to satisfy the requirement of the public transportation in cities, so as to promise commercial profits. Therefore, industries have to solve such manufacturing challenges, while, fortunately, modern manufacturing techniques from the automotive business can serve as a meaningful reference to significantly reduce the costs of making even complex flying cars by exploiting the integration of new high-tech composites, battery technology, and alloys. Similar to the automotive industry, making flying cars affordable to common people will definitely accurate the popularization and commercialization process of FCTS in both private and public transportation markets.

2) Pilot Training \& Hiring: Though autonomous driving techniques have been developed for fly cars by equipping self-piloting model, pilots still cannot be completely deprived of FCTS, at least in near future, even when costs of hiring and training pilots, the salary of which is typically higher 
than that of a taxi driver, can be saved. Because the role of human pilots cannot be taken place for some safety reasons in some emergency circumstances. To elaborate, so far, similar to self-driving cars, public perception is not strong enough as people are not totally confident with self-piloting techniques. Then, the commercialization of the flying cars with fully selfpiloting will be a long process, though the authors believe it will happen eventually. Thus, it can be concluded that pilot training and hiring will lead to an ineluctable operation cost to FCTS.

3) Commercial Licence Application: Undoubtedly, commercial licenses must be applied for commercializing FCTS not only from the economic authorities of the central government but also from the civil aviation regulation department, e.g., commercial space transportation licenses from Federal Aviation Administration of the United States and European Union Aviation Safety Agency. Furthermore, pilot certificates must be applied from and granted by the government to approve the competence in handling flying cars under all potential flying cases, which is similar to traditional air flights. Therefore, plenty of efforts must be devoted to promoting and realizing large-scale commercialization of FCTS, due to the business competitions with traditional transportation systems and the exploration of the NGS over the urban area.

\section{Ethical Issues}

As FCTS operates in the NGS, there are some possible ethical issues incurred by the VOL and flying behaviors of flying cars, such as disturbances to the ecosystem, invasions of human privacy, and pollution to the environment.

1) Disturbances to Ecosystem: Because the height of the operation space for FCTS normally ranges from tens of meters to hundreds of meters, which is the main living space of the birds, irresponsible and wanton operations of fly cars will cause some negative impacts on the birds' lives, i.e., disrupting nests, provoking attacks, scattering leks, interrupting feeding, and midair collisions. Furthermore, the noise coming from the frequent TOL and flying behaviors of flying cars will also bring influences to on-ground animals. Though these potential risks may exist, there are several ways that can positively harness the negative effects of FCTS, such as monitoring sensitive areas, poaching records, habitat preservation, etc. Then, this collected ecosystem information is taken into account during the design and updating process of flying routines, so as to weaken/minimize all potential damages to the ecosystem and make FCTS be eco-friendly.

2) Invasions of Human Privacy: Another problem arisen from the flying behaviors of flying cars is the invasions of human privacy. Imagine that flying cars are operating over urban areas or residential areas, people's activities will be prone to be exposed, observed and supervised. Likewise, attention must be paid while designing and updating the routines for flying cars in public applications. On the other hand, the flight bans considering spatial planning should be approved to protect sensitive areas, like the airspace above private properties or prohibited zones.
3) Environmental Pollution: The main pollution brought by FCTS is inadequately controlled noise from the flying operations presents a growing danger to the health and welfare of the population, particularly in urban areas. Therefore, the commercialization of FCTS should obey the noise regulations proposed by the government, e.g., the Noise Control Act from the environmental protection agency of the United States [37]. Secondly, another pollution is introduced by FCTS: If the flying cars frequently fly around the airspace above the urban areas, a lot of visual pollution will appear to the urban landscape. In order to avoid this, flying routines should be carefully designed and planned.

Furthermore, as discussed in Section III, the main power trend of flying cars is electric type, and then power batteries are necessary for FCTS, which are charged from the grid. Then, there exist two pollution problems indirectly resulted from FCTS as follows.

1) Environmental pollution caused by batteries: Since battery production causes more environmental damage than carbon emissions alone, and the used batteries contain toxic heavy metals, new technologies of green battery production, and advanced recycling techniques of waste batteries are required.

2) The emissions during electricity generation: Though today new renewable energy is the fastest-growing energy source (e.g., solar, wind, geothermal and modern biofuel production), $76 \%$ of all electricity on the grid across the world and $90 \%$ in the United States in 2018 are generated by traditional biofuels including petroleum, natural gas, or coal [38]. Therefore, the centralization of the energy source of FCTS via power batteries still indirectly consumes considerable hydrocarbon fuels, calling for green power generations on the country/world level to cut down emissions.

\section{CONCLUSION}

In this paper, We have presented a comprehensive study on FCTS in five focused subject areas. First, we reviewed the developing history of flying cars in view of both timeline and the technique categories. Second, we discussed and compared the main existing designs of flying cars from four aspects: TOL modes, pilot modes, operation modes, and power types. Third, we also introduced the designs of FCTS by respectively considering path and trajectory planning, supporting facilities and commercial designs. Moreover, the challenges and future developing directions of FCTS are provided in detail, respectively.

Based on the discussions and comparisons among the main existing designs of flying cars, some general guidelines for the future design and manufacture of flying cars can be addressed as follows:

- Emergence mechanism must be prepared and safety equipment must be installed on flying cars, as the safety of passengers is the first and most important thing for commercialization of flying cars;

- Flying cars should be robust to operate in certain undesirable weather cases;

- VTOL and electric power are necessary for the flying cars operating in urban areas, due to the adaptability 
to space-constrained urban areas and the environmental friendliness;

- Flying cars with HTOL is a good choice for some special application scenarios with enough space to build runways;

- Hydrocarbon Fuel and hybrid power are suitable for the fly cars operating in long-distance transportation;

- Autonomous pilot is the best choice for the flying cars operating along with fixed lines and time schedule, which is similar with the cases in public bus and train services, due to the saved operation costs on fuel and pilot;

- Similar to airplanes, the hybrid pilot mode is a better choice for normal flying cars, because of the combination of the liberation of pilots and the human safety backup in emergence cases.

Furthermore, according to the presented discussions on the designs and challenges of FCTS, some useful principles for the construction and commercialization of FCTS are highlighted as follows:

- Collision/obstacle avoidance mechanism must be designed for FCTS, while search \& rescue program must be predefined before the commercialization of FCTS;

- Green manufacture and operation should be encouraged and advocated in FCTS to avoid any unnecessary environmental pollution;

- New and accurate position and navigation algorithms are required for FCTS, which operates in 3D space, other than traditional grounded transportation systems working in two-dimensional space;

- The path/trajectory planning should be conducted and optimized for specific operation scenarios, while considering some constraints, e.g., weather conditions and conservation spaces;

- The communication system for FCTS should be specialized to promise the coverage performance in 3D NGS, while seriously taking the information security problem into consideration during the design of the control signal system for FCTS;

- The locations of TOl sites and SMF should be jointly chosen, emerged, and optimized to realize the global optimal in specific serving areas, as the functions of TOL and SMF can be launched in same physical space;

- The main market opportunity for FCTS is public human transportation, which can greatly release the pressure of traffic congestion in urban areas, while there are also some specific application scenarios for FCTS requiring specialized on-demand services;

- Manufacturing and operation cost should be controlled and saved to promise the survival of FCTS in the market competitions with traditional transportation systems;

- More efforts must be made to receive the supports not only from the investors but also from the government, so as to respectively achieve the financial and policy supports, and further to guarantee the successful commercialization of FCTS.

\section{REFERENCES}

[1] M. Wall, Flying car technology is turning a dream into reality, BBC News, Oct. 31, 2013.
[2] G. H. Curtiss, Convertible aircraft, US Patent, No. US1294413A, Feb. 4, 1917.

[3] J. Meaden, "The Waterman aeroplanes," Air Britain Archives, no. 3, pp. 81, 1998.

[4] Flying auto crashes; lands in California mud flats - pilot is only bruised, The New York Times, pp. 29, Nov. 19, 1947.

[5] S. Gilmore, "Tired of the commute? All you need is $\$ 3.5$ million," The Seattle Times, Nov. 3, 2016.

[6] Taylor Aerocar III, Museum of Flight. Retrieved: March 4, 2012.

[7] Car that can fly away developed in Van Nuys, The Van Nuys News, May 15, 1973.

[8] J. J. Corn, B. Horrigan, Yesterday's tomorrows: Past visions of the American future, Johns Hopkins University Press, Baltimore, Maryland, United States, May 1996.

[9] Piasecki tests twin-turbine and seagoing VTOLs, Aviation Week \& Space Technology, vol. 76, no. 19, p. 83, May 1962.

[10] B. Zoltan, Boeing sky commuter is a flying car concept, Carscoops, July 6, 2015, Available: https://www.carscoops.com/2015/07/boeing-skycommuter-is-flying-car/

[11] https://www.pal-v.com

[12] https://www.uber.com/us/en/elevate/

[13] https://kittyhawk.aero/

[14] https://www.airbus.com/innovation/urban-air-mobility/vehicledemonstrators/vahana.html

[15] https://www.aeromobil.com/

[16] https://www.volocopter.com

[17] https://www.moog.com/innovation/aircraft/SureFly.html

[18] https://www.opener.aero/

[19] https://terrafugia.com/

[20] https://www.ehang.com/

[21] https://wisk.aero/

[22] https://www.jobyaviation.com/

[23] https://www.jauntairmobility.com/

[24] https://www.boeing.com/features/frontiers/2019/autonomous-flyingvehicles/index.page

[25] https://en.skydrive2020.com/

[26] https://www.astonmartin.com/en-us/models/future-models/the-astonmartin-volante-vision-concept-usa

[27] https://evtol.news/aircraft/moller-skycar-m400/

[28] Bell reveals a surprisingly down-to-earth air Taxi, Wire, Jan. 7, 2019. Available: https://www.wired.com/story/bell-nexus-air-taxi-flying-car/

[29] https://detroitflyingcars.com/

[30] http://www.deloreanaerospace.com/

[31] https://www.audi.com/en/experience-audi/mobility-and-trends/emobility/blue-sky-thinking.html

[32] Are flying cars preparing for takeoff? Morgan Stanley, Jan. 23, 2019. Available: https://www.morganstanley.com/ideas/autonomous-aircraft

[33] Bus Maintenance Facility Working Group, Architectural and engineering design for a transit operating and maintenance facility, American Public Transportation Association, Sept. 2011.

[34] The JD Drone program, available: https://corporate.jd.com/whatIsNew Detail? contentCode $=6 \mathrm{IhXLeeSAFLjLLlyuZatDA}$

[35] Prime Air, available: www.amazon.com

[36] K. Rajashekara, Q. Wang and K. Matsuse, "Flying cars: Challenges and propulsion strategies," IEEE Electrification Magazine, vol. 4, no. 1, pp. 46-57, Mar. 2016.

[37] Clean Air Act Title IV - Noise Pollution, Environmental Protection Agency, United States, 1990, available: https://www.epa.gov/clean-airact-overview/clean-air-act-title-iv-noise-pollution

[38] https://www.c2es.org/content/renewable-energy/ 